\title{
SOLUTION APPROACHES TO DIFFERENTIAL EQUATIONS OF MECHANICAL SYSTEM DYNAMICS: A CASE STUDY OF CAR SUSPENSION SYSTEM
}

\author{
Tesfaye O. Terefe ${ }^{1}$, Hirpa G. Lemu ${ }^{2}$ \\ 1 School of Mechanical Engineering, Jimma University, 378 Jimma, Ethiopia, e-mail: tesfitti2016@gmail.com \\ 2 Faculty of Science and Technology, University of Stavanger, N-4036 Stavanger, Norway, e-mail: Hirpa.g.lemu@uis.no
}

Received: 2018.02.09

Accepted: 2018.04.22

Published: 2018.06.01

\begin{abstract}
Solution of a dynamic system is commonly demanding when analytical approaches are used. In order to solve numerically, describing the motion dynamics using differential equations is becoming indispensable. In this article, Newton's second law of motion is used to derive the equation of motion the governing equation of the dynamic system. A quarter model of the suspension system of a car is used as a case and sinusoidal road profile input was considered for modeling. The state space representation was used to reduce the second order differential equation of the dynamic system of suspension model to the first order differential equation. Among the available numerical methods to solve differential equations, Euler method has been employed and the differential equation is coded MATLAB. The numerical result of the second degree of freedom, quarter suspension system demonstrated that the approach of using numerical solution to a differential equation of dynamic system is suitable to easily simulate and visualize the system performance.
\end{abstract}

Keywords: Numerical method, Euler method, Differential equation, Suspension system, MATLAB, Mechanical system modeling.

\section{INTRODUCTION}

Numerical methods tend to emphasize the implementation of algorithms and provide systematic methods for solving problems on computers by numerical calculations in a tabular data (approximate data) or graphical representation and figures. The process of solving problems generally involves starting from an initial data, using high precision digital computers, following the steps in the algorithms, for obtaining the results and the methods are approximate ones [12].

For all systems undergoing change, differential equations are used to properly describe the system dynamics. The use of differential equations is crucial in engineering, science and other areas like economics and related fields. Nowadays, there are many techniques available for solving differential equations, which were devel- oped through many years of studies particularly in mathematics. Solving differential equations based on numerical methods of approximation started before programmable computers existed. For large and complex system, in particular, differential equations are suitable for solutions employing numerical methods that are simulated in computers. This is because numerical simulations can represent the physical system and used to find the nearly accurate solutions, which are not possible analytically.

In the mechatronics system design and analysis, in particular, mechanical systems, even including thermal or chemical nature, are exploited with analogies to electrical systems to design and analyze [8]. The developments in programmable computers have also made it possible to solve complex systems of differential equations by writing simple program codes on a personal computer. 
Thus, simulation of differential equation using diverse methods and techniques in a numerical approximation became popular.

For vehicle dynamics in general and the suspension system in particular, numerical method based solution of differential equations has been widely implemented [10]. The automobile industry has closely studied, and still interested in, the vehicle suspension system because it is the mechanism that physically separates the vehicle body from the wheels of the car to improve the ride stability, comfort and road handling of vehicles. To better understand the suspension system performance, therefore, studies focusing on description of the road surface, modelling of the suspension control system, identification of the performance criteria and the like are reported [14]. The vehicle is commonly modelled and simulated using the properties of its components such as springs, shock absorbers and linkages that connect the vehicle to its wheels. Generally, the traditional suspension system consists of springs and dampers that are referred to as passive suspension, while latter types that are externally controlled are referred to as semi-active or active suspension systems. The suspension stores energy in the spring and dissipates through the damper. Some design approaches for vehicle suspension systems focus on unconstrained optimizations for suspension system to identify the desirability of low suspension stiffness, reduced unsprung mass, and an optimum damping ratio for the best controllability [2]. Others use numerical methods to partially automate the suspension system design process using genetic algorithm (GA) employing a global search technique $[3,9,11]$. The mathimatical modeling and optimization of the suspension system is mostly described by differental eqautions. For instance, Thoresson and colleagues [18] proposed a methodology that performs optimization of off-road vehicle suspension system using gradient based approach. Goga and Klucik [6] have used evolutionary computation method to optimize the vehicle suspension system by developing a half-car model in MATLAB.

The objective of the study reported in this article is to make a closer investigation of the application of numerical solution to a differential equation of mechanical system dynamics with focus on vehicle suspension system dynamics as a case study. Upon reviewing the previous scientific works related to this area and exploring the methods of numerical solution to the differential equation as well as the concept of suspension system, a mathematical model of a selected suspension system is described using differential equations. The application of the most popular numerical method, such as Euler method to differential equation of vibration analysis are studied and the solution proposals are simulated using MATLAB tool using ode45 programming algorithm. The governing differential equation of the motion of a quarter suspension system is derived using Newton's Second Law.

Following this introduction section, the basics of numerical solution approaches of differential equations and different types of suspension systems in vehicles are briefly presented. Then, a case study mathematical modelling approach is illustrated and the differential equations that describe the vehicle dynamics are derived. After discussing the results of the modelling done in MATLAB, using plots of the simulation outputs, the article finally summarizes the content in the conclusion section.

\section{DIFFERENTIAL EQUATIONS AND NUMERICAL METHODS}

Differential equations are used to model and describe problems that involve changes of some variables (exchanges of matter, energy, information or any other quantities, often as they vary in time and/or space) with respect to another. In many real life situations, differential equations that model the problem are too complex to solve by integration exactly. However, there are different categories of differential equations for a specific problem type. A differential equation that is classified as ordinary differential equation contains one independent variable and its derivative. Many mechanical systems are influenced by several unknown variables and their derivatives in which a differential equation category referred to as partial differential equation may be properly used. Furthermore, most real world problems are nonlinear, and hence, linearization is often used to transform the problem into a linear system and model by using a linear differential equation. This equation has one degree of freedom of unknown function and its derivatives. Further categories exist depending on their degree or order, i.e. classifications as first order, second order, higher order or n's degree of (multi-degree) of differential equations.

Solving differential equations analytically is difficult and tedious, even if the solution exists. 
As analytical solution of most of differential equations is unknown, applying the numerical methods is necessary. On the other hand, in contrast to the general analytical solutions of initial value problems that can be adapted to any initial conditions, numerical method belongs to specific initial value problem that changes the initial value in an iterative calculation process. As Thohura and Rahman [17] explained in their comparative study, different techniques of numerical approaches are available for solving differential equations including powerful numerical analysis software packages. For vibration analysis, in particular, numerical approaches are to be used if the free or forced vibration of a system cannot be integrated in closed form for the differential equation governing the system. The numerical methods employed for this purpose include the finite difference method, central difference method, Euler method, the fourthorder Runge-Kutta method and stiff differential equation method [12]. These methods are based on the approximation of the derivatives appearing in the equation of motion and the boundary conditions are used for solving differential equations in numerical method.

\section{SUSPENSION SYSTEM AND ITS COMPONENTS}

A suspension system is the main part of vehicles that can ensure the tire contact with the ground surface. It connects the vehicle chassis and wheels physically, transmits all loads from the ground to vehicle body, and enables the vehicle steering, braking and driving systems. It consists of wheels and tire, springs and shock absorbers and linkages [14].

The suspension system is one of the most important systems in a vehicle because it ensures driving comfort by the vertical motion of the vehicle, which depends on the quality of the contact between the tire and ground surface [1].

Suspension systems are mainly classified as passive, semi-active and active, and these categories are briefly explained in the following subsections.

\section{Passive suspension system}

A passive suspension system, i.e. a system without energy source [7] is one of the conventional systems containing springs and shock absorbers (Fig. 1). As illustrated, this suspension system has been commonly modelled with two degrees of freedom $\left(\mathrm{x}_{1}\right.$ and $\left.\mathrm{x}_{2}\right)$ for the motion of

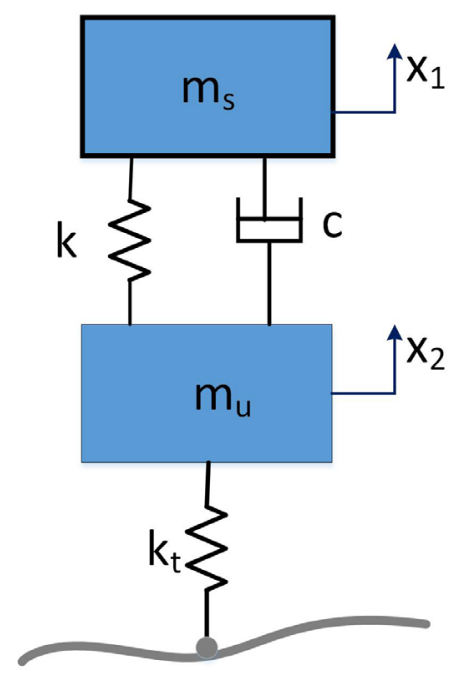

Fig. 1. Illustration of passive suspension system

the sprung $\left(\mathrm{m}_{\mathrm{s}}\right)$ and unsprung $\left(\mathrm{m}_{\mathrm{u}}\right)$ masses supported by the suspension system. Considering a quarter model of a car, the constants $k$ and $k_{t}$ represent the stiffness models of the suspension spring and the tyre stiffness respectively.

While springs have linear or nonlinear characteristics, shock absorbers (dampers) exhibit nonlinear behavior [20] in terms of the relationship between force and relative velocity. Although this type of suspension system does not fulfill all expectations about comfort and safety, it is widely used because it is one of the simplest designs and hence is cheap.

\section{Semi-active suspension system}

This suspension system type (Fig. 2) contains, instead of passive shock absorber, an active shock absorber that is automatically controlled by an integrated regulator. Because of this device, the suspension system becomes semi-active and it offers some advantages in case of extreme driving conditions $[13,19]$. Earlier versions of semi-active suspension systems were regulated by adjusting the resistance to fluid flow through oil orifices, which is too slow for the speed of the mechanical motion [21]. Latter applications, however, use electrorheological and magnetorheological fluids, which are smart materials made by mixing fine particles into a low viscosity liquid [4]. To study the performance of semi-active suspension systems, several models are developed and reported in the literature [16]. Compared with the fully active system the semi-active suspension requires less energy, it is cheaper and it is simplest in design. 


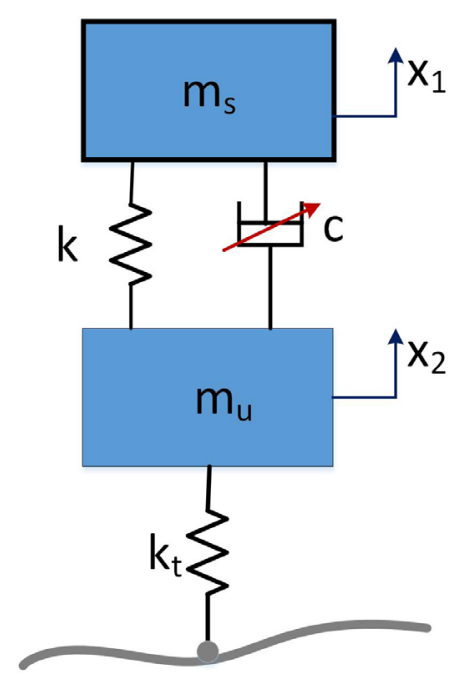

Fig. 2. Illustrative model of a semi-active suspension system

\section{The fully active suspension system}

The fully active suspensions system (illustrated in Fig. 3), beside normal components, contains also an actuator, sensors and central processing unit (CPU). Sensors measure the acceleration and the programmable CPU computes and guides the actuator that produces the additional forces when desired. Thus, this suspension system has better benefits over the other types because of its adaptability to the road profile while driving. However, the passive system is more widely used because of the simplicity and comparable lower weight [13].

The purpose of all the suspension system types briefly presented above is to isolate the

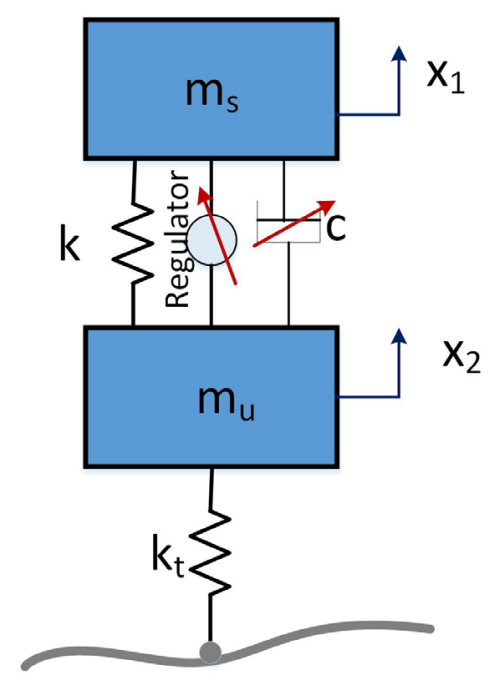

Fig. 3. Illustration of active suspension system forces arising from the road by ensuring high level of manageability and convenience.

The car motion exposes it to a multitude of dynamic forces such as aerodynamic forces, car vibrations, weather conditions and surface roughness induced vibrations. These vibrations are harmful to the passenger with a negative impact on the experienced comfort. A suspension system, thus, must meet several functions including: comfort, safety and handling. To fulfil these and other requirements, it is necessary to minimize the vertical acceleration and vertical relative displacement between sprung mass and wheel, and keeping vibration levels of the suspension system below the threshold imposed by the established natural frequency [15].

\section{MATHEMATICAL MODELLING OF VEHICLE SUSPENSION SYSTEM}

A mathematical model is a description of a system using equations. Differential equations constitute a major field of study in mathematics with wide applicability in problems of engineering in mechanical, electrical circuits, civil engineering etc. Among others, the evolution of processes that are deterministic, differentials and dimensional finite are studied. In this article, a mathematical model is used to describe and study the performance of the suspension using a system of two simple differential equations of order two.

Figure 4 shows a model of the quarter of a car suspension system. To study the dynamic performance of the model, the free body diagram of the quarter of the car mass (sprung mass) $\mathrm{m}_{1}$ and the mass of the wheel-tire-axle assembly (unsprung mass) $m_{2}$ are shown in Fig. 4(b).

The spring constant $\mathrm{k}$ represents the elasticity of the suspension spring and the spring $k_{t}$ represents the elasticity of the tire. $x_{0}(t)$ is the displacement input due to the surface irregularity of the road. The actuator force, F, applied between the sprung and unsprung masses is controlled by feedback and represents the active components of the suspension system [5].

In this model, it is assumed that the mass $m_{1}$ moves faster than the mass $\mathrm{m}_{2}$, and the elongation of the spring $\mathrm{k}$ is $\mathrm{x}_{1}-\mathrm{x}_{2}$. The force exerted by the spring $\mathrm{k}$ on the mass $\mathrm{m}_{1}$ is downward, as it tends to restore to the unreformed position. According to Newton's third law, the force exerted by the spring $\mathrm{k}$ on the mass $\mathrm{m}_{2}$ has the same magnitude, but opposite in direction. Other spring forces and 


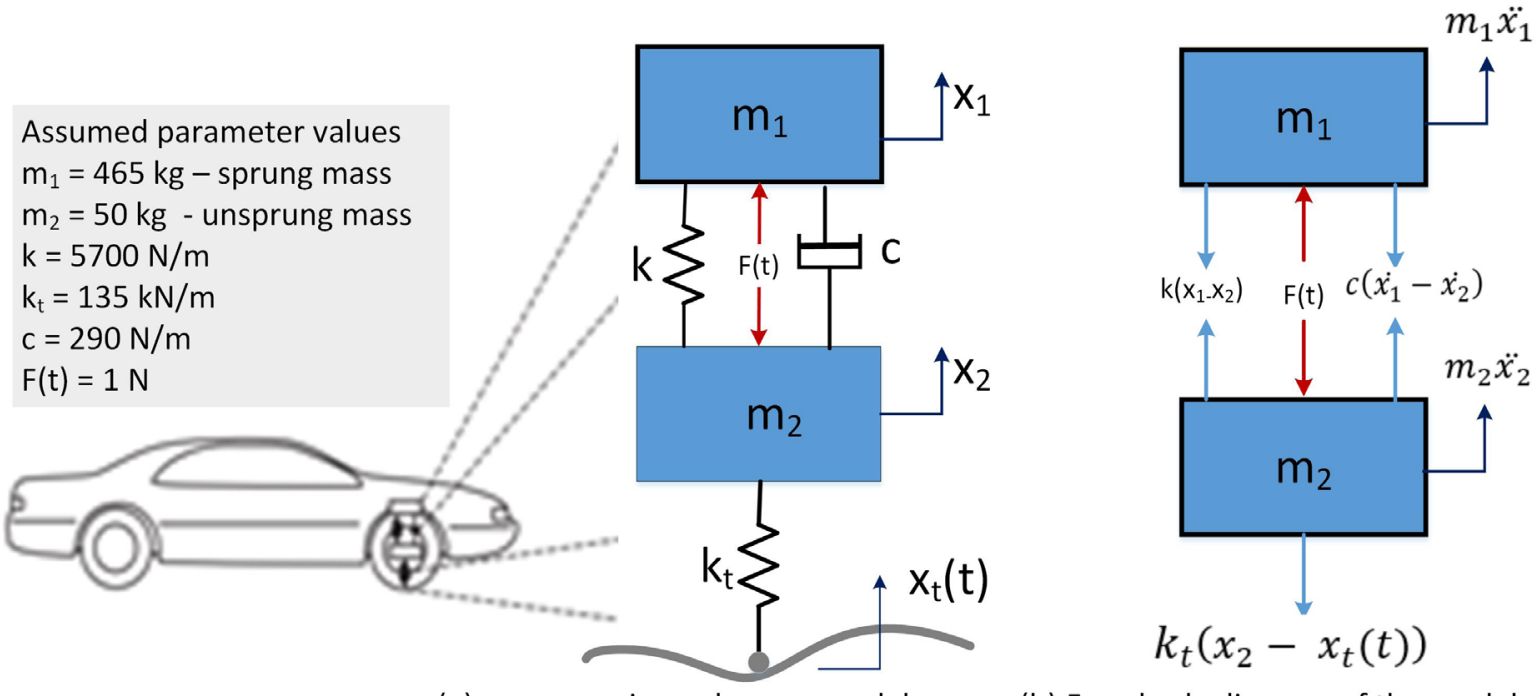

(a) mass - spring - damper model

(b) Free-body diagram of the model

Fig. 4. Simulation model of quarter car

damping forces can be determined using the same logic. The gravitational forces are not included in the free-body diagrams in Fig. 4.

Applying Newton's second law, the equations of motion for the displacement $x_{1}$ and $x_{2}$ are expressed as:

$$
\begin{gathered}
F-k\left(x_{1}-x_{2}\right)-c\left(\dot{x_{1}}-\dot{x_{2}}\right)=m_{1} \ddot{x}_{1} \\
-F+k\left(x_{1}-x_{2}\right)+c\left(\dot{x_{1}}-\dot{x_{2}}\right)-k_{t}\left(x_{2}-x_{t}(t)\right)=m_{2} \ddot{x_{2}}
\end{gathered}
$$

Rearranging the equations into the standard input - output form

$$
\begin{gathered}
m_{1} \ddot{x_{1}}+c\left(\dot{x_{1}}-\dot{x_{2}}\right)+k\left(x_{1}-x_{2}\right)=F \\
m_{2} \ddot{x_{2}}-k\left(x_{1}-x_{2}\right)-c\left(\dot{x_{1}}-\dot{x_{2}}\right)+k_{t} x_{2}=-F+k_{t} x_{t}
\end{gathered}
$$

This can be expressed in the second order differential equation form as

$$
\left[\begin{array}{cc}
m_{1} & 0 \\
0 & m_{2}
\end{array}\right]\left[\begin{array}{l}
\ddot{x}_{1} \\
\ddot{x_{2}}
\end{array}\right]+\left[\begin{array}{cc}
c & -c \\
-c & c
\end{array}\right]\left[\begin{array}{l}
\dot{x}_{1} \\
\dot{x_{2}}
\end{array}\right]+\left[\begin{array}{cc}
k & -k \\
-k & k+k_{t}
\end{array}\right]\left[\begin{array}{l}
x_{1} \\
x_{2}
\end{array}\right]=\left[\begin{array}{cc}
1 & 0 \\
1 & k_{t}
\end{array}\right]
$$

where a sinusoidal input $x_{t}(t)=\sin (t)$ for the road profile is considered for the analysis.

\section{Numerical Solution: Euler Method}

As mentioned earlier in this article, the mathematical models of the dynamic system are expressed in terms of differential equations (Eqn. 5). In particular, state-space representations take the form of sets of first-order differential equations, where numerical solutions of these equations are implemented by numerical integration of the first derivatives of the state variables. Therefore, the numerical solutions of the responses of dynamic systems such as the one formulated above are obtained by numerical integrations.

Among the diverse methods of numerical approach to solve the differential equation, Euler's method, modified Euler's method, Runge-Kutta method and stiff methods are those widely used in mechanical system modelling and simulation. Euler methods for differential equation is selected and employed for this case study as the approach of numerical solution to the governing equation of motion of the quarter car suspension.

Eigen values and Eigen vectors of a system are key elements for solving frequencies and mode shapes of dynamic systems. For large order systems of multi-degree of freedom, computing the Eigen values is more difficult and tedious because of its higher order. The space state representation helps to reduce the degrees of the higher orders of equation to the first order of differential equation. Using Euler 


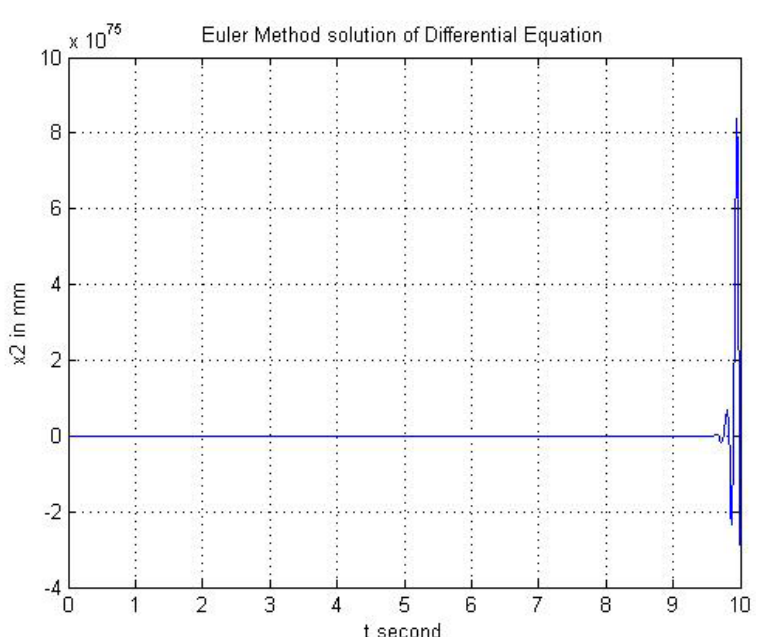

Fig. 5. Displacement $x_{1}$ response for sprung mass

method as a numerical method to solve differential equations, the space state form of damped multidegree of freedom of a vibration of the automobile quarter suspension model is expressed as a forced response of a damped linear system as:

$$
\mathrm{M}(\ddot{\mathrm{x}})+\mathrm{C}(\dot{\mathrm{x}})+\mathrm{K}(\mathrm{x})=\mathrm{F}(\mathrm{t})
$$

To solve the formulated differential equation (Eqn. 5) using Euler method, simple MATLAB program is coded and simulated. The response in terms of the displacement of the sprung and unsprung masses is plotted in Figs. 5 and 6.

\section{Numerical Simulation of the Time Response}

To find the numerical solution of the state space form, the ode45 function in MATLAB software was used, a code which examines the use of numerical integration to simulate and plot the response of a vibration of the dynamic system.

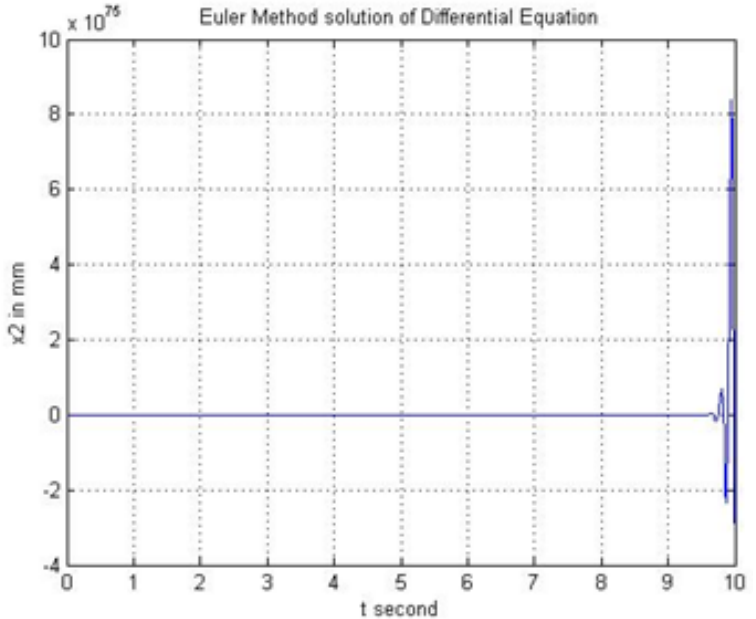

Fig. 6. Displacement $x_{2}$ response for unsprung mass

As demonstrated, simulation is much easier way to obtain the systems response when compared, for example, to computing the response by modal decomposition. The modal decomposition approach is needed to perform design of the dynamic system, to get insight into the dynamics of the system, and to check numerical solutions. Likewise, the numerical solution may also be used to check analytical works.

\section{BRIEF DISCUSSION OF RESULTS}

The response of suspension system for the sprung and unsprung masses are plotted in Fig. 7 and Fig. 8 respectively. The response of car body oscillation (Fig. 8) shows that the system is stable but needs some time to settle down and the settling time is moderate. The vibration will be only transparent to the passenger during the settling

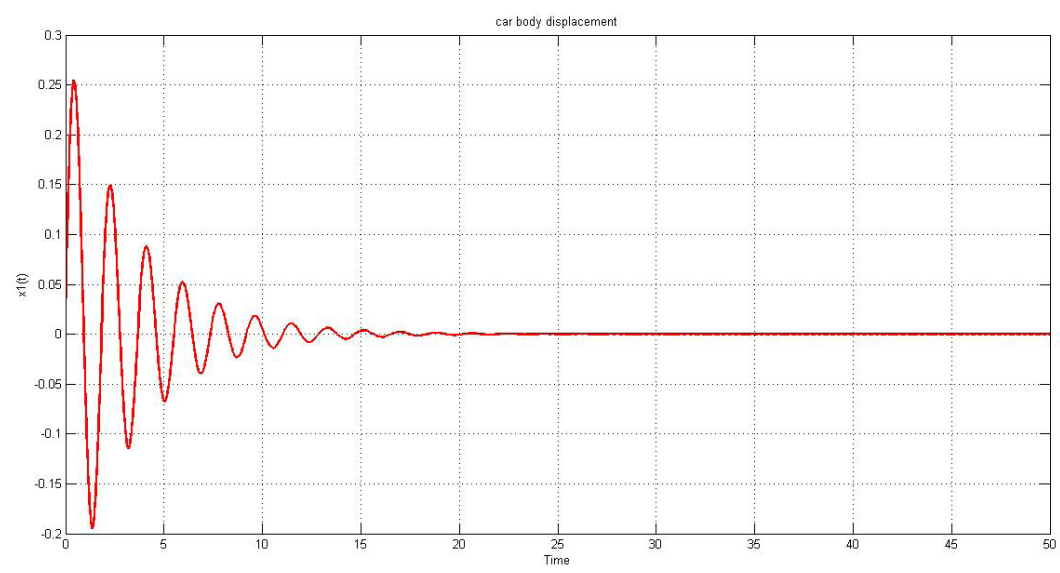

Fig. 7. Response of car body displacement (sprung mass) 


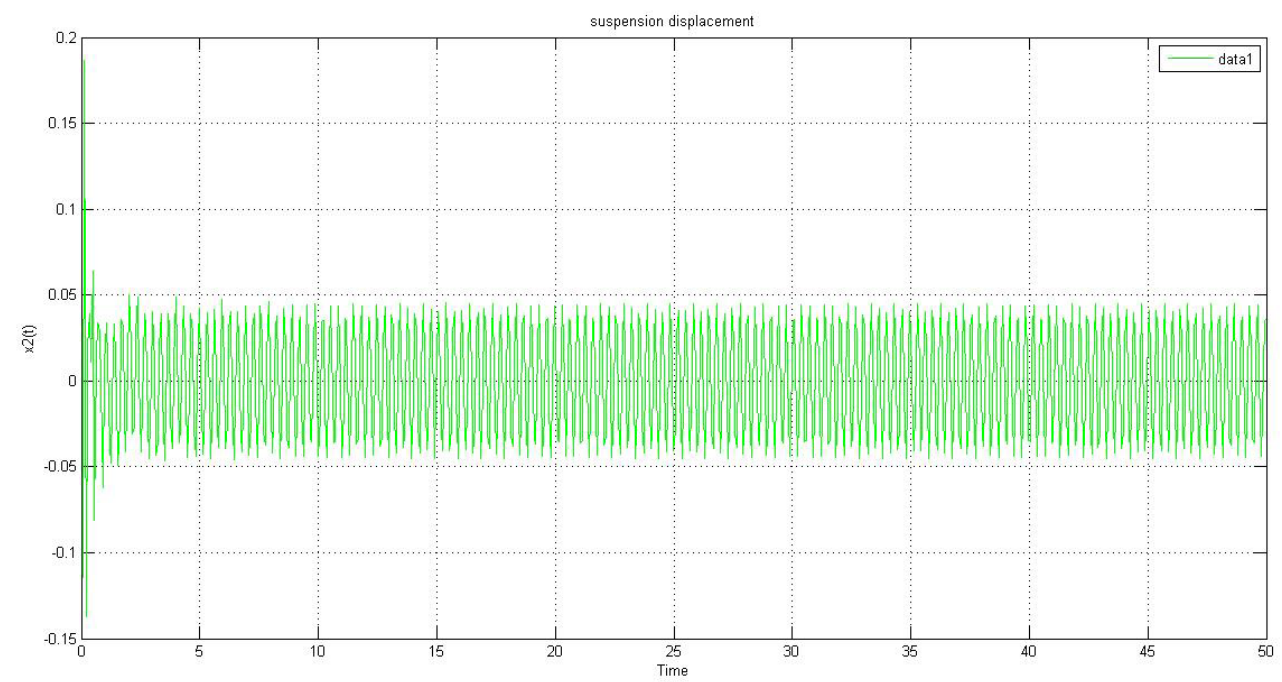

Fig. 8. Response of the suspension system (unsprung mass)

time. This means the passengers sitting in the automobile will feel some amount (approx. 30\% of the total time taken for this simulation) of vibration at the starting of the car, it goes decreasing gradually and becomes stable. In order to eliminate or reduce vibration at the settling time a feedback controller can be added into the system to improve the performance of the suspension. This allows the suspension system exhibit good comfort by dissipating the vibration energy through the damper.

As can be observed from the response plot in Fig. 8, the suspension system is vibrating highly at the initial time (very short time with high vibration) and then comes to a normal sinusoidal motion in which the damper can dissipate out the vibration from the system to prevent it passing to the passenger or to the sprung mass.

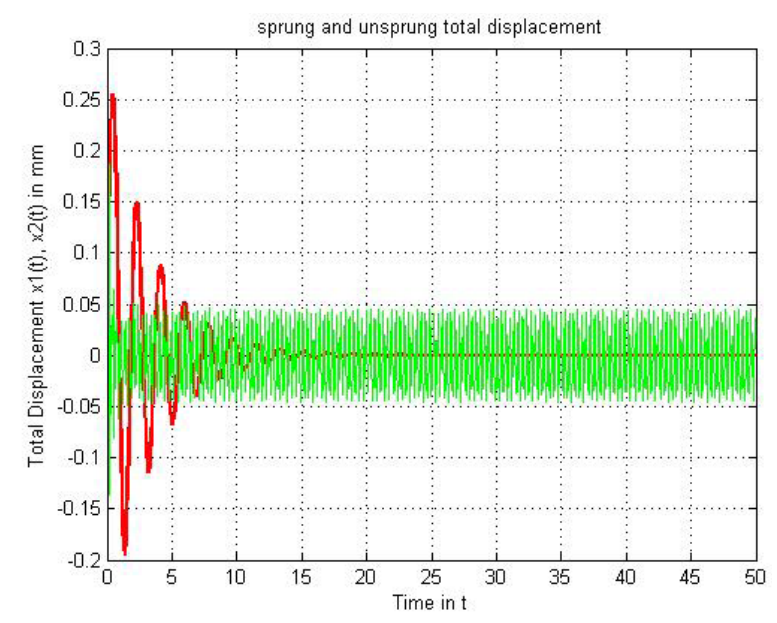

Fig. 9. Overall system response
The overall system response (Fig. 9) shows that the suspension system (unsprung mass) is subjected to uniform vibration after settling time. This indicates that improvement measures to the system is required in order to provide a comfortable, safe travel and to extend the service lifetime of suspension system.

\section{CONCLUSION}

This article presented a study conducted on a dynamics (mechanical) system simulation approach using numerical methods. The system, specifically the suspension system of a car, is expressed by differential equations and numerical method is used to solve by programming in MATLAB. In particular, the ode 45 algorithm is used to simulate the system model and observe the response or the dynamic condition of the system.

The contribution of this work is primarily educational, especially in the field of Applied Mechanics and Dynamic system of mechanical engineering. The modelling approach using differential equations and the solution approach employing Euler method are demonstrated. The simulation outputs given in plots of system response contribute to better understanding of the system performance.

\section{REFERENCES}

1. Allen, R., Magdaleno, R., Rosenthal, T., Klyde, D. et al., Tire modeling requirements for vehicle dynamics simulation, SAE Technical Paper 950312, 1995, https://doi.org/10.4271/950312. 
2. Alleyen, A. and Hedrick, J. K., Nonlinear adaptive control of active suspensions, IEEE Trans. Control Systems, 3, 1995.

3. Baumal, A.E. McPhee, J.J. and Calamai, P.H., Application of genetic algorithms to the design optimization of an active vehicle suspension system, Computer Methods in Applied Mechanics and Engineering, 163 (1-4), 1998, 87-94.

4. Boelter, R. and Janocha, H., Performance of longstroke and low-stroke MR fluid damper. In: Proceedings of SPIE 3327, Smart Structures and Materials 1998: doi: 10.1117/12.310693.

5. Esfandiari, R.S., Modeling and analysis of dynamic system, 2nd ed. USA, NY, CRC press, 2014.

6. Goga, V. And Klucik, M., Optimization of vehicle suspension parameters with use of evolutionary computation, Proceedia Engineering, 48, 2012, 174-179: doi.org/10.1016/j.proeng.2012.09.502.

7. Jayachandran, R. and Krishnapillai, S. Modeling and optimization of passive and semi-active suspension systems for passenger cars to improve ride comfort and isolate engine vibration, Journal of Vibration and Control, 19(10), 2012, 1471-1479: doi: $10.1177 / 1077546312445199$.

8. Karnopp, D.C., Margolis, D.L. and Rosenberg, R.C., System dynamics: modeling, simulation, and control of mechatronic systems, 5th ed., 2012, John Wiley \& Sons, NJ.

9. Khajavi, M.N., Notghi, B. And Paygane, G., A multi objective optimization approach to optimize vehicle ride and handling characteristics, International Scholarly and Scientific Research \& Innovation., 4 (2), 2010, 502-506.

10. Kortüm, W. DLR., Review of multibody computer codes for vehicle system dynamics, Vehicle System Dynamics, 22(1), 1993, 3 - 31.

11. Mitra, A.C., Desaib, G.J., Patwardhan, S.R., Shirke, P.H., Waseem M.H. Kurne Nilotpal Banerjee, Optimization of Passive Vehicle Suspension System by Genetic Algorithm, Procedia Engineering, 144, 2016, 1158-1166. https://doi.org/10.1016/ j.proeng.2016.05.087.

12. Rao, G.V., Rao, T.R.M., Rao, K.S. and Purushottam,A., Analysis of passive and semi-ac- tive controlled suspension systems for ride comfort in an omnibus passing over a speed bump, International Journal of Research and Reviews in Applied Science, 5, 2010.

13. Sharp, R. S. and Hassan, A. S., The relative performance capabilities of passive, active and semiactive car suspensions, Proceedings of Institution of Mechanical Engineers 20(D3), 1986, 219-228.

14. Sharp, R.S. and Crolla, D.A., Road vehicle suspension system design - a review, Vehicle System Dynamics, 16(3), 1987, 167 - 192.

15. Shirahatti, A., Prasad, P.S.S. Panzade, P. and Kulkarni, M.M., Optimal design of passenger car suspension for ride and road holding, Journal of Brazilian Society of Mechanical Science and Engineering, vol. 30(1). http://dx.doi.org/10.1590/ S1678-58782008000100010.

16. Spencer, B.F., Dyke, D.J., Sain, M.K. and Carlson, J,D., Phenomenological model of a magnetorheological damper. Journal of Engineering Mechanics, 123(3), 1997, 230-238.

17. Thohura, S. and Rahman, A., Numerical approach for solving stiff differential equations: A comparative study, Global Journal of Science Frontier Research Mathematics and Decision Sciences, 13, 2013.

18. Thoresson, M.J., Uys, P.E., Els, P.S. and Snyman, J.A., Efficient optimisation of a vehicle suspension system using a gradient-based approximation method, Part 1: Mathematical modelling. Mathematical and Computer Modelling, 50, 2009, $1421-1436$.

19. Verros, G., Natisiavas, S. and Papadimitriou, C., Design optimization of quarter-car models with passive and semi-active suspensions under random road excitation, Journal of Vibration and Control, 11, 2005, 581-606.

20. Wallaschek, J., Dynamics of non-linear automobile shock-absorbers, International Journal of Non-Linear Mechanics 25, 1990, 299-308.

21. Yao, G.Z., Yap, F.F., Chen, G., Li, W.H. and Yeo, S.H., MR damper and its application for semi-active control of vehicle suspension system, Mechatronics, 12, 2002, 963-973. 